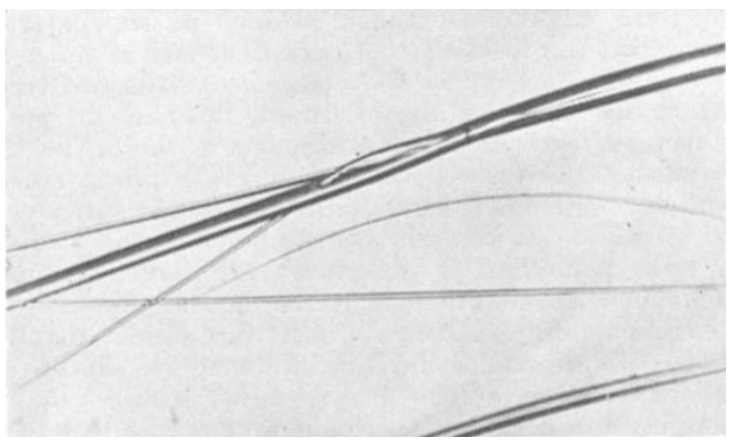

Fig. 5. Forking and interpenetration of hydroquinone crystal. $\times 130$. (Photograph by $R$. W. Gooding)

microns thick have been observed to break spontaneously under no bending strain, an effect presumably produced by high internal stress.

High strength is not of course confined exclusively to crystals which have been grown in the bent state.
Fig. 4 shows a hydroquinone crystal a few mierons thick grown straight and afterwards bent to about 5 per cent strain by mechanical manipulation in petroleum jelly.

Not very much is known about the nature of the defects which weaken the thicker crystals. Since these crystals can be grown bent and will almost always become fully straightened when released, it is unlikely that the defects are of the nature of edge dislocations. Films which have been taken of the growth of the thicker crystals show that complicated forking and rejoining mechanisms such as that seen in Fig. 5 undoubtedly occur, but it is doubtful if these account for all the facts. It is not easy to postulate a growth mechanism by which substantial defects can be introduced without at the same time introducing the probability of a relief of strain energy which would result in a 'permanent set' in crystals grown while bent.

This article is published by permission of the Chairman of Tube Investments, Ltd.

${ }^{1}$ Hardy, H. K., "Progress in Metal Physics", 6, 45 (1956).

2 Menter, J. W., Proc. Roy. Soc., A, 236, 119 (1956).

\title{
SUB-MICROSCOPIC ORGANIZATION AND FUNCTION OF NERVE CELLS
}

\begin{abstract}
N international symposium on "The Fine A structure of the Nervous System" was held in the Venezuelan Institute for Neurology and Brain Research during March, under the auspices of the International Society for Cell Biology. The proceedings will be published as a supplement to Experimental Cell Research. The papers were drawn from the fields of electro-physiology, electron microscopy, chemistry and histochemistry. Spectroscopy and flame photometry also figured in the programme. Inspection of the Institute during the Symposium showed it to be the best equipped in the world for the application of physical techniques to the study of biological problems. Probably the most intriguing new technique demonstrated was that of nuclear magnetic resonance and its application to the measurement of rapid changes in the water content of untreated intact nerve. Nuclear magnetic resonance is a form of spectrometry, and it provides a new technique for the study of magnetic and paramagnetic properties of elements. 'The method depends upon the principle of precessing the elementary magnetic moments of atomic nuclei in a homogeneous field and then causing transitions between the various quantum states by the application of radio-frequency magnetic fields. A proton magnetic resonanco signal is then produced and can be recorded: from it the water content of the tissue can be determined. The principle has many applications apart from the estimation of water, and ultimately it should be possible to determine free radicals and the content of a variety of chemical compounds including ribonucleic acid in nerve and other tissues. This technique is at present being applied to neuro-biological studies only at the Venezuelan Institute for Neurology and Brain Research. Physicists may know that the technique of nuclear magnetic resonance is to be used, in conjunction with the Earth satellite to be released
\end{abstract}

later in the year, to measure the Earth's magnetic field at great altitudes.

The papers at the symposium fell into four groups : on the nerve fibres, the nerve cell membrane, the neuron, and the receptors. The papers presented in the first of these groups centred around the theme of axon-satellite cell relationships, and this naturally included the structure of the myelin sheath. Dr. Herbert Gasser was concerned particularly with the structure of unmedullated nerves. He established as a general principle that non-medullated fibres run in, and are supported by, the Schwann sheaths of medullated fibres in mixed nerves. Dr. Gasser pointed out that the sustentacular cells and basal cells of the olfactory mucosa behave like Schwann cells in this aspect, and he demonstrated a very intimate relation between the basal cells and the axon of the olfactory cell passing between them. This led naturally to the consideration of the relationship between satellite cells and the myelin sheath of medullated nerves. Dr. F. O. Schmitt dealt with the recent suggestion, based on experimental observations on neurogenesis in tissue culture, that the satellite cell wraps in spiral fashion around the axon, thus producing alternate layers of phospholipid and protein (the jelly-roll theory); and Dr. Fernandez Moran's beautiful high. resolution electron micrographs demonstrated that the laminæ of the myelin sheath which he had previously described could indeed be traced in a spiral pattern. Dr. Moran also demonstrated that if nerve is centrifuged at $200,000 \mathrm{~g}$ for twenty-four hours with its axis radially arranged, the axonal material is centrifuged into a drop. By this means pure axon material can be obtained for investigation. Under such treatments the lamination of the myelin sheaths is well preserved. The myelin sheath was the subject of X-ray diffraction studies by Dr. J. B. Finean, and he showed that a distinction must be made between myelin of peri- 
pheral nerves and myelin of central origin. He also demonstrated differences due to dehydration and em. bedding with methacrylate as compared with gelatin.

Prof. A. von Muralt showed that during excitation of nerve, bound thiamine is liberated as free thiamine and that this is split very rapidly into at least two substances, $X_{1}$ and $X_{2}$, which are resynthesized into thiamine during recovery if energy from glycolysis is available. 'These thiamine transitions are closely connected with the sodium transport system, and Dr. $P$. Mueller, by burning a stimulated nerve fibre in a flame photometer, demonstrated an appreciable gain in sodium, and that this was equal to the loss of potassium which also occurred. He found that 10 per cent of the potassium in nerve cannot be exchanged in this way. It is of interest that Dr. Lorente de Nó found that sodium ions are not necessary in the external medium of nerve fibres for the conduction of action potentials, but that on the other hand the action potential is directly related to the amount of sodium inside the nerve fibre. He also demonstrated that a number of onium ions can be substituted for sodium and can restore the ability to conduct impulses in the sodium-deficient frog. His results indicate that local anæsthesia is due to competitive inhibitions of chemical reactions which require the presence of sodium inside the nerve fibre.

Dr. C. G. Bernhard showed in model experiments with cats and monkeys that corresponding doses of local anæsthetics selectively block the ephaptic type of neuron interaction, and that small intravenous doses of local anæsthetics thus reduce the ephaptic motorsensory fibre transmission at the injured region of peripheral nerve. Dr. J. Folch Pi directed attention to the fact that nervous tissue contains ionic lipids, for example, phosphatidyl serine, diphosphine inositide and cerebron sulphuric acid. Whother such lipids are related to the ionic balance in nervous tissue has yet to be discovered.

Dr. G. H. Bourne pointed out that a number of phosphatases are concentrated along the membranes of the dendrites of the pyramidal cells in the cerebral cortex and that these and other cells and their processes appear to be embedded in phosphatase-rich tissue. Attention was also directed to the fact that the basal cells of the olfactory epithelium, with which the axons of the olfactory cells enter into intimate contact, show extremely high phosphatase activity. In a review of modern theories of ion transport in membranes, Dr. T. T'eorell suggested that the mutual action of electrochemical and hydrostatic 'pressure' differences might lead to oscillatory membrane phenomena revealed by periodic variations in membrane potential, membrane conductance and pressure difference.

From cell processes interest then turned to the neurone itself, and Prof. R. Jung showed how extracellular recordings of single-neuron discharges from the visual cortex in the cat 'encephale isolé' preparation were used for investigating the co-ordination of the cortical neuronal system. He found that the neurons of the visual cortex show a well co-ordinated pattern following light stimuli and a tendency to restrain excitation. He concluded that this neuronal system tends to maintain a medium level of activity and an adequate regulation of cortical function corresponding to an actual situation by four self-regulating control mechanisms. This was followed by Dr. D. P. I. Lloyd's paper on "The Approach of Unitary Analysis to Neuron Response". He described how as each motoneuron encountered is examined, it is charac- terized according to the number of responses in a set number of trials at afferent stimulation fixed in frequency and intensity. 'This assemblage of units represents a synthetic motoneuron pool and the sum of their individual behaviours can be compared in a given situation with that of a natural pool. This synthetic pool can be utilized to provide information that cannot be extracted from a study of natural pool responses. Such a study of individuals suggests that details of heterosynaptic organization, rather than wide variation of motoneuron threshold, underlies heteronymous transmission during the peak of post-tetanic potentiation, and that low-frequency depression is due to action upon the motoneurons rather than to a reduction in presynaptic efficacy. Dr. Lloyd directed attention to the fact that the introduction of the intracellular microelectrode tech. nique for representative sampling of motoneurons would appear to merit the most careful attention. His paper was therefore very appiopriately followed by Dr. G. Svaetichin's paper on action potential analyses and electrical stimulation of single neurons, in which he demonstrated that when an electrical stimulation was applied on to the perikaryon of a dorsal root ganglion cell, either extra- or intracellularly, the action potential always started at the $M$-region of the axon, and he suggested that in synaptic activation the electrical response also starts in the same $M$-trigger region. Further papers on the neuron dealt with its chemistry and its fine structure as seon with the electron microscope. Dr. H. Hydén described methods in use in his laboratory for the localization and quantitative determination of ribonucleic acid nucleotides, lipids and protein in nerve cells. Dr. V. L. Van Breemen described the presence of small vesicles in the axon, visible under the electron microscope, and found that these and other constituents undergo similar changes in both neuron and axon following ligation of the axons or injection of malan nnytryl. Dr. Fernandez Moran demonstrated the characteristic association of membrane profiles with the dense granular material of the nerve cell body, the presence of filament-like structures in the cytoplasm, the fine structure of the nucleolus and pores in the nuclear membrane 700-800 A. across. Dr. J. L. Luft showed a system of 'electroplaques' in the electric tissue of fishes which contain a highly developed system of tubules and vesicles extending from the plasma membrane into the interior of the plaque. This system could be associated with one phase of ion transport occurring by vesicular transfer, in a manner analogous to that proposed for the function of synaptic vesicles in nerve endings.

These synaptic vesicles were beautifully demonstrated by Dr. E. de Robertis and Dr. S. L. Palay in their respective papers; and also in adrenal medulla and neurohypophysis by the latter. Dr. de Robertis dealt with the possibility that these vesicles contained acetyleholine. Dr. S. Kuffler discussed synaptic inhibitory mechanisms and gave an analysis of inhibitory processes which took place at the isolated two-neuron synapse between the dendrites of the sensory cell and an inhibitory axon. Prof. G. Causey deseribed changes in the synaptic areas in the supərior cervical ganglion following pre- and post-ganglionic section, and Prof. T. H. Bulloch gave an account of synaptic and intra-neural parametery of integration in some invertebrate ganglion cells. Dr. R. Couteaux demonstrated the localization of acetylcholine in motor end plates. 
The final section of the conference dealt with the receptors. Dr. Fermandez Moran described the structure of the insect retina, which is of particular interest in view of the ability of insects to orient themselves by polarized light. Dr. George Wald summarized the relation of visual pigments to the process of vision, and Dr. G. Svaetichin gave an analysis of the receptor potentials from single cones. Dr. E. F. MacNicholl gave an account of the subliminal slow potentials recorded from the eye of Limulus, and Dr. H. Autrum an electrophysiological analysis of the visual systems of insects. Drs. Forbes and Deane presented electrical studies of colour vision in the turtle. The physiology of olfactory, sound and taste receptors was described by Drs. D. Ottoson, H. Engstrom and Y. Zotterman. Drs. K. Porter and G. Palade discussed membrane-limited systems, in particular the endoplasmic reticulum in the cytoplasm, and paid special attention to its manifestations in striated muscle, where it was suggested it may play a part in transmitting depolarization from the sareolemma to the myofibrils.
Membrane resting potential of muscle was considered by Dr. J. W. Woodbury, the morphology of muscle fibres with different conducting properties by Dr. H. Ruska and ciliary co-ordination in Protozoa by Dr. L. E. Roth. Dr. L. U. C. Junquiera gave a statistical account of the number of nerve cells and ganglia in the different parts of the intestinal plexuses, and Prof. J. Runnstrøm analysed the cytoplasinic changes occurring upon fertilization in the sea urchin egg.

It is obvious that a wide field was covered, and all taking part were agreed that the symposium was a considerable success. On the Saturday following the conclusion of the symposium, the President of Venezuela visited the Institute, met the participants and spent most of the morning talking with them. There is no doubt that the Venezuelan Institute for Neurology and Brain Research has been scientifically launched and that it is embarking upon a series of fundamental investigations on the nervous system which cannot fail to make it the outstanding Institute of its kind in the world. Geoffrey H. Bourne

\section{CYTO-TAXONOMY}

$\mathrm{T}$ HE Linnean Society of London held a wholeday session on cyto-taxonomy on May 3. Dr. W. B. Turrill introduced the symposium. He pointed out that the study of chromosomes was only one branch of cytology, and expressed a hope that cell contents other than the nucleus would receive due consideration. There is no doubt that cytological data can often be used by the taxonomist, but they have to be correlated with characters from many other branches of botany and zoology and taxonomically evaluated in connexion with these. Insufficient numbers of species have so far been adequately studied cytologically, especially in tropical and subtropical floras, for wide taxonomic generalizations validly to be made on the basis of cytology. Many taxonomists are whole-heartedly in favour of synthetic taxonomy and welcome any contribution that cytologists can make towards elucidating the problems of taxonomy. The number of biologists who regard cytologists as queer and perverse ultra-modernists who "peer down a microscope and count their own eye-lashes" is dwindling. They are, however, irritated by those cytologists who, from ignorance, scorn the basic work of taxonomists and appear to believe that scoring the chromosomes tells one everything on $\theta$ needs to know about a plant. Exaggerated claims or extreme criticisms made by taxonomists or by cytologists are to be deplored. What is needed is cooperation based on an understanding of each other's aims and methods and expressed in attempts to synthesize results.

Mr. J. L. Hamerton, in his paper, "Problems in Mammalian Cytotaxonomy", began by reviewing the advances in technical methods which had occurred during the past ten years and which had led to the development of modern techniques for the study of mammalian chromosomes, so that rapid and accurate chromosome counts, and detailed studies on chromosome morphology were now possible. It was emphasized that for comparative work counts of chromosome number alone were not sufficient and that studies should embrace all aspects of chromosome morphology.

The concept that the chromosome number must necessarily be constant throughout the range of a morphological species was discussed and rejected, and it was pointed out that it was the population which was of major importance in cytogenetical work rather than the morphological species.

The relative importance of aneuploidy, polyploidy and structural change was considered in respect of mammalian evolution, and it was emphasized that there was not necessarily any direct relationship between chromosomal and morphological change, though there are certain obvious exceptions to this. Mr. Hamerton considered that the main result of chromosomal change was the introduction of discontinuity leading to some degree of isolation or variation which in turn might lead to eventual phenotypic differences of a specific nature.

The results of chromosomal change in mammalian evolution were considered at both the inter-specific and intra-specific levels. Data on experimental polyploidy in the mouse were reviewed briefly, and the evidence for polyploid evolution in the hamsters was discussed. It was considered that insufficient evidence was available to determine whether polyploid evolution had occurred here or not. Further detailed morphological studies on the chromosomes were required, and some attempt should be made to produce hybrids and back-crosses, perhaps by artificial insemination, for subsequent cytological analysis.

Chromosomal polymorphism and the existence of chromosome races or cryptic species in mammals was discussed, and their evolutionary significance considered.

Dr. A. J. Cain stated that although there is general agreement that chromosomes are important in taxonomy there has been little analysis of what is meant by taxonomic importance. The study of chromosomes can contribute three main classes of 